\title{
Interface Structure-Property Relations through Aberration-Corrected STEM
}

S.J. Pennycook, ${ }^{*}$ A.Y. Borisevich, ${ }^{*}$ M. Varela, ${ }^{*}$ A.R. Lupini, ${ }^{*}$ H.J. Chang, ${ }^{*}$ D.N. Leonard, ${ }^{*}$ T.J. Pennycook, ${ }^{*, * *}$ M.P. Oxley, ${ }^{*, * *}$ J.C. Idrobo, ${ }^{*, *}$ H. Yurdakul, ${ }^{*, * *}$ S. Turan, ${ }^{* * *}$ P. Yu, ${ }^{* * * *}$ R. Ramesh ${ }^{* * * *}$ and S.T. Pantelides. ${ }^{* * * *}$

*Materials Science and Technology Division, Oak Ridge National Laboratory, Oak Ridge, TN, USA

** Department of Physics and Astronomy, Vanderbilt University, Nashville, TN, USA

${ }_{* * * * *}^{* *}$ Materials Science and Engineering Department, Anadolu University, Eskisehir, Turkey

**** Department of Materials Science and Engineering and Department of Physics, University of

California, Berkeley, CA, USA

The aberration-corrected STEM provides quantitative information on atomic positions, species and chemical bonding with new levels of precision and sensitivity. Multiple signal channels are available, some simultaneously, including the high-angle ADF signal, the phase contrast signal with an axial detector, an annular bright field signal [1] and spectroscopic images. Such data can be directly compared with density functional calculations to reveal the atomistic origins of interfacial properties.

Complex oxide interfaces present the most challenging systems, with many possible structural distortions and mixed valence electronic systems. With an aberration-corrected STEM, good quality bright field phase contrast imaging becomes possible, allowing quantitative measurement of column locations, including light elements [2]. Figure $1 \mathrm{~A}$ shows a bright field image of a $\mathrm{BiFeO}_{3}$ $\mathrm{La}_{0.7} \mathrm{Sr}_{0.3} \mathrm{MnO}_{3}(\mathrm{BFO} / \mathrm{LSMO})$ interface obtained with a VG Microscopes HB603U STEM operating at $300 \mathrm{kV}$. Light $\mathrm{O}$ columns can be seen as small dark spots between the larger dark spots of the cation columns [3]. From the angles between adjacent $\mathrm{O}$ column positions the local octahedral tilts can be determined, after correction for local image distortion by comparison to the cation-cation angles. In this way an accurate map of octahedral tilts can be extracted, as shown in Fig. 1B. The checkerboard nature of the tilts is quite apparent, as expected from the bulk structure, shown schematically in Fig. 1C. The magnitude of the tilts is progressively suppressed near the interface with LSMO, as shown in Fig. 1C, which is expected to influence local electronic properties. Indeed, the interface region shows up in EELS as having electronic properties different from either BFO or LSMO [2]. Quantitative measure of lattice parameter is also possible directly from the Z-contrast image, and the same region shows an anomalous lattice expansion normal to the substrate [3].

Spectroscopic imaging provides an alternative means to measure octahedral tilts. Although signal levels are lower, atomic-resolution maps can now be obtained with high efficiency. Figure 2 shows an image of $\mathrm{LaMnO}_{3}$ in the [010] zone axis orientation obtained with a Nion UltraSTEM operating at $60 \mathrm{kV}$. Comparing the elemental images of the $\mathrm{O}$, La and $\mathrm{Mn}$ edges, the octahedral rotations of the $\mathrm{O}$ sublattice are very clear. $\mathrm{O}$ atoms in the $\mathrm{LaO}$ column are invisible at this specimen thickness due to the strong depletion of the probe as it propagates down the column [4].

Equally challenging are amorphous/crystal interfaces, since light atom positions cannot be seen in amorphous materials at today's resolutions. However, information on heavy atom positions can be extracted quite simply, as shown in Fig. 3. In all these examples, theoretical modeling is the key to linking observed atomic and electronic structures to interfacial properties. 
References:

[1] H. Rose, Optik 39, 416 (1974), S. D. Findlay et al., Appl. Phys. Lett. 95, 191913 (2009)

[2] K. Urban, Science 321, 506 (2008)

[3] arXiv:1002.2989v1 [cond-mat.mtrl-sci]

[4] M.P. Oxley et al., these proceedings

[5] This work was supported by DOE Basic Energy Sciences, Division of Materials Science and Engineering, and by appointment to the ORNL Postdoctoral Research Program administered jointly by ORNL and ORISE (HJC).

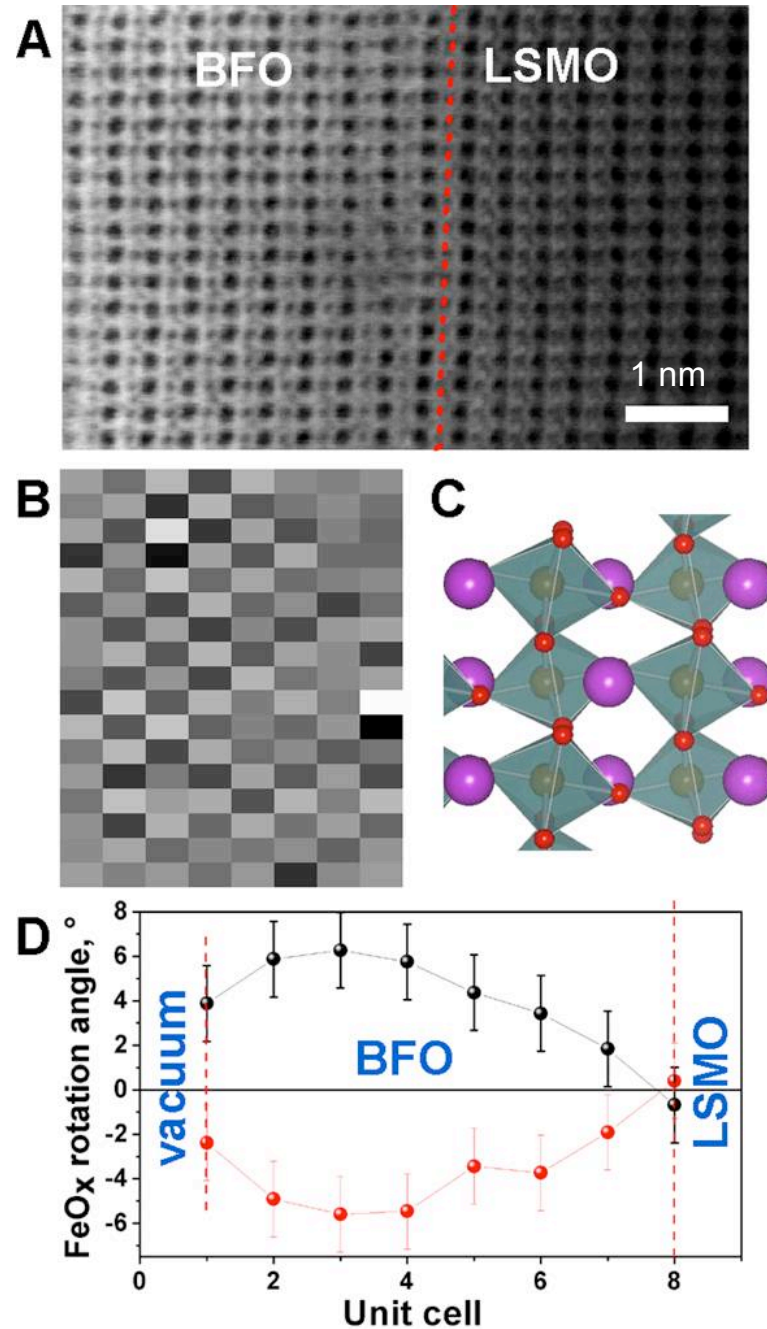

Fig. 1: (a) Bright field image of a BFO/LSMO ultrathin film. (b) 2D map of in-plane octahedral rotation angles in $\mathrm{BFO}$ showing checkerboard order. (c) BFO structure in rhombohedral (001) orientation showing the expected tilt pattern. (d) line profile obtained from the map in (b); error bars are set to the standard deviation of local Bi-Bi angles. Scale bar is $1 \mathrm{~nm}$. Reproduced from [3].
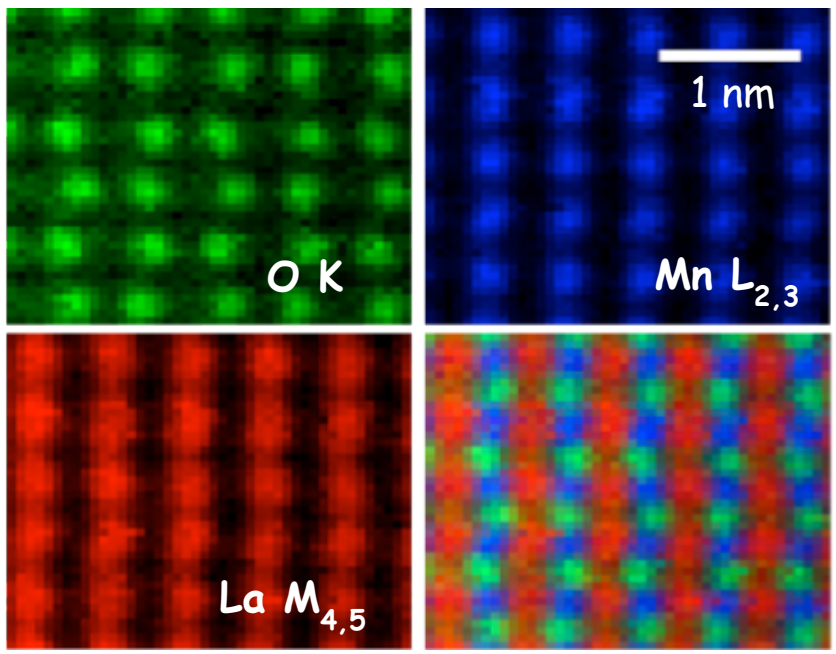

Fig. 2: Spectroscopic imaging of $\mathrm{LaMnO}_{3}$ along the [010] zone axis using the $\mathrm{O} \mathrm{K}, \mathrm{La} \mathrm{M}_{4,5}$ and $\mathrm{Mn} \mathrm{L}_{2,3}$ edges in green, blue and red, and a composite image. Octahedral rotations of the $\mathrm{O}$ sublattice are visible. Images are corrected for scan distortions.

Fig. 3: ADF image of [0001] $\beta$-SiAlON doped with $\mathrm{Yb}_{2} \mathrm{O}_{3}$ and $\quad \mathrm{CeO}_{2} \quad$ showing segregation of rare earth atoms to the intergranular film. Possible $\mathrm{Yb}$ and $\mathrm{Ce}$ sites are shown in blue and yellow, $\mathrm{Si}$ in red and $\mathrm{N}$ in green and dark blue.

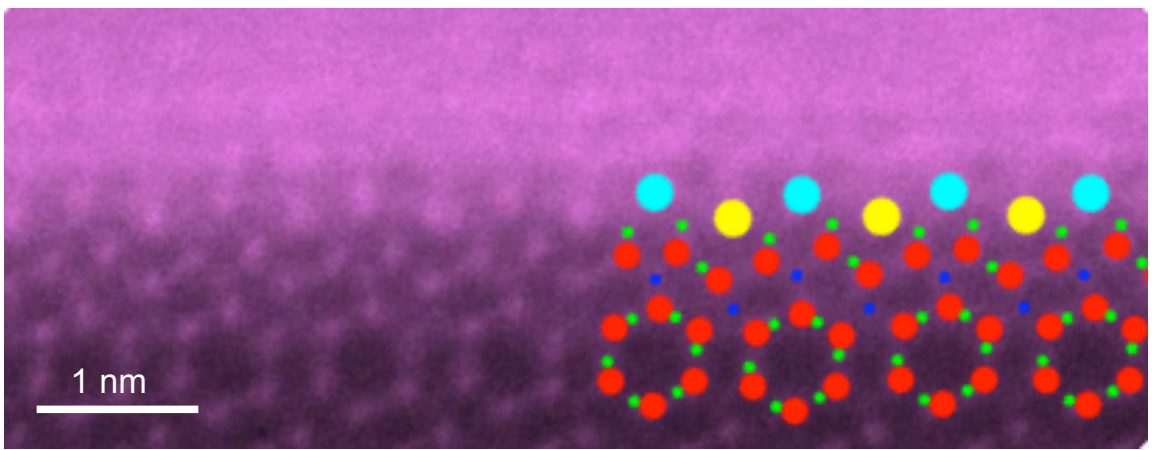

